\title{
Size Does Matter. Sterically Demanding Metallocene-Substituted 3-Methylidene-Oxindoles Exhibit Poor Kinase Inhibitory Action
}

\author{
John Spencer, ${ }^{*,+}$ Jahangir Amin, ${ }^{+}$Peter Coxhead, ${ }^{\perp}$ John McGeehan, $^{\perp}$ Christopher J. Richards, \\ Graham J. Tizzard, ${ }^{\neq}$Simon J. Coles, ${ }^{\ddagger}$ John P. Bingham, ${ }^{\S}$ John A. Hartley, ${ }^{\S}$ Li Feng, Eric Meggers, ${ }^{\#}$ and \\ Matthew Guille ${ }^{\perp}$ \\ ${ }^{+}$School of Science, University of Greenwich at Medway, University of Greenwich, Chatham, ME4 4TB, U.K. \\ ${ }^{\ddagger}$ UK National Crystallography Service, School of Chemistry, University of Southampton, Highfield, Southampton, SO171BJ, U.K. \\ ${ }^{\S}$ CRUK Drug-DNA Interactions Research Group, UCL Cancer Institute, Paul O'Gorman Building, 72 Huntley Street, London, WC1E 6DD, U.K. \\ ${ }^{\perp}$ School of Biological Sciences, University of Portsmouth, King Henry Building, Portsmouth, PO1 2DT, U.K. \\ "School of Chemistry, University of East Anglia, Norwich, NR4 7TJ, U.K. \\ \#Fachbereich Chemie, Philipps-Universität Marburg, Hans-Meerwein Strasse, D-35032 Marburg, Germany
}

Supporting Information

ABSTRACT: 1,3-Dihydro-2H-indol-2-one (1) undergoes microwave-mediated Knoevenagel condensations with 1,2,3,4,5pentaphenylferrocene carboxaldehyde $(\mathbf{2 b})$ or $\left(\eta^{4}\right.$-tetraphenylcyclobutadiene $)\left(\eta^{5}\right.$-cyclopentadienylcarboxaldehyde $)$ cobalt (2c) to afford metallocene-substituted 3-methylidene-1,3-dihydro- $2 \mathrm{H}$-indol-2-one $\mathbf{4}$ or $\mathbf{5}$, respectively, in excellent yields as separable E,Z-stereoisomeric mixtures. Both $\mathbf{4}$ and $\mathbf{5}$ exhibited poor kinase inhibition and no appreciable cytotoxicity toward a leukemia cell line, attributed to the steric bulk of the metallocene substituents.
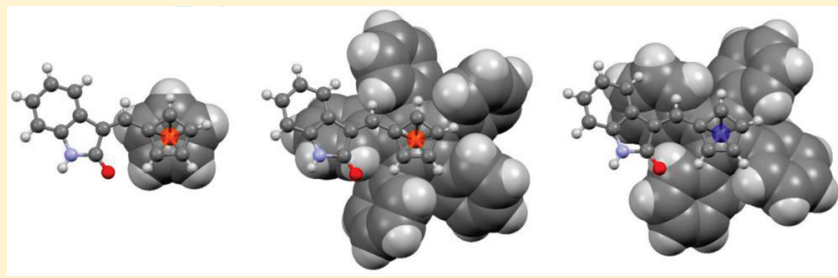

\section{INTRODUCTION}

Metallodrug design is a burgeoning field of bioorganometallic chemistry with applications spanning therapeutic agents to biological tools, probes, and diagnostics. ${ }^{1}$ Recent progress in the area has identified transition metal-based agents where the architectural topology, by virtue of the higher coordination numbers accessible to the biologically inert transition metal, can impart outstanding selectivity and potency toward targets such as kinases, which are often aberrant in cancer. ${ }^{2} \mathrm{We}$ and others recently reported a Knoevenagel condensation of 1,3-dihydro- $2 \mathrm{H}$-indol2-one (2-oxindole) (1) with ferrocenecarboxaldehyde (2a), which yields a mixture of $(E)$ - and $(Z)-3$, which are readily separated by chromatography. Both isomers were characterized in the solid state and display substantial kinase inhibitory action, notably as VEGFR-2 (vascular endothelial growth factor receptor-2) inhibitors. ${ }^{3}$ Following this study, structural-activity relationship studies (SAR) have been carried out on 3 aiming to optimize the biological activity of these molecules; hence, $\mathrm{N}$-methylation, substitution on the aryl ring by amino or bromo substituents, hydrogenation of the exocyclic double bond, and replacement of the ferrocene by ruthenocene have been performed, and excellent kinase inhibition was observed, notably against DYRK3 and 4 (dual-specificity tyrosine-phosphorylation-regulated kinases). ${ }^{4}$ We now report the synthesis of oxindoles substituted by metal complexes containing bulky ligands (Scheme 1 ), ${ }^{5}$ in order to study their solution and solid-state properties as well as their
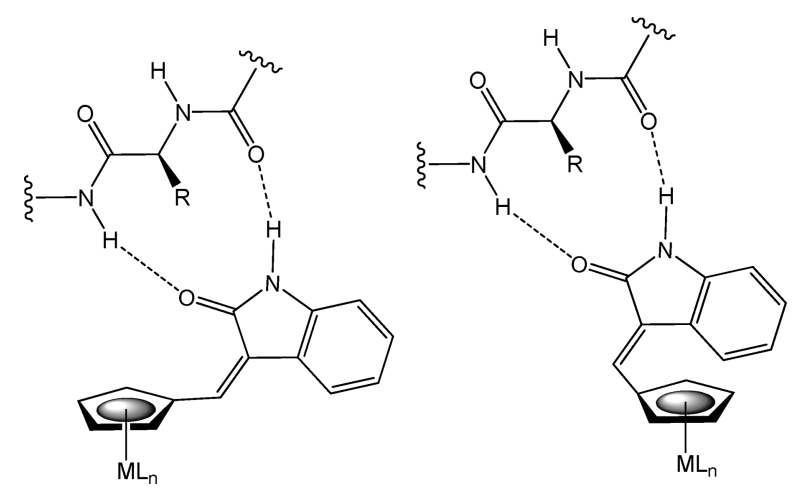

Figure 1. Anticipated binding mode for oxindole complexes in the hinge region of kinases.

biological activity since changing the metal center may have a profound effect on the biological activity of the resulting complex especially in terms of its kinase binding (Figure 1).

Microwave-mediated $(\mu \mathrm{W})$ condensation reactions are routine in organic synthesis, and not surprisingly a number of kinase inhibitors have been synthesized by this methodology; however, the use of microwaves in organometallic synthesis is much less

Received: March 28, 2011

Published: May 11, 2011 
Scheme 1. Knoevenagel Condensations of 1 with 2<smiles>O=C1Cc2ccccc2N1</smiles>

1

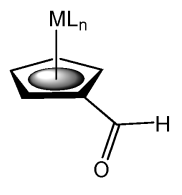

2

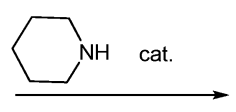

$\mathrm{EtOH}, \mu \mathrm{W}$.

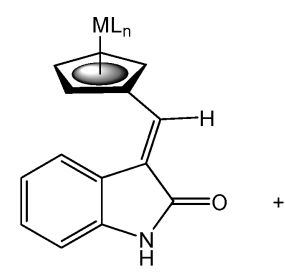

(E)-3-5<smiles>N#[Y14]C1C=CC(C=C2C(=O)Nc3ccccc32)C1</smiles>

$(Z)-3-5$
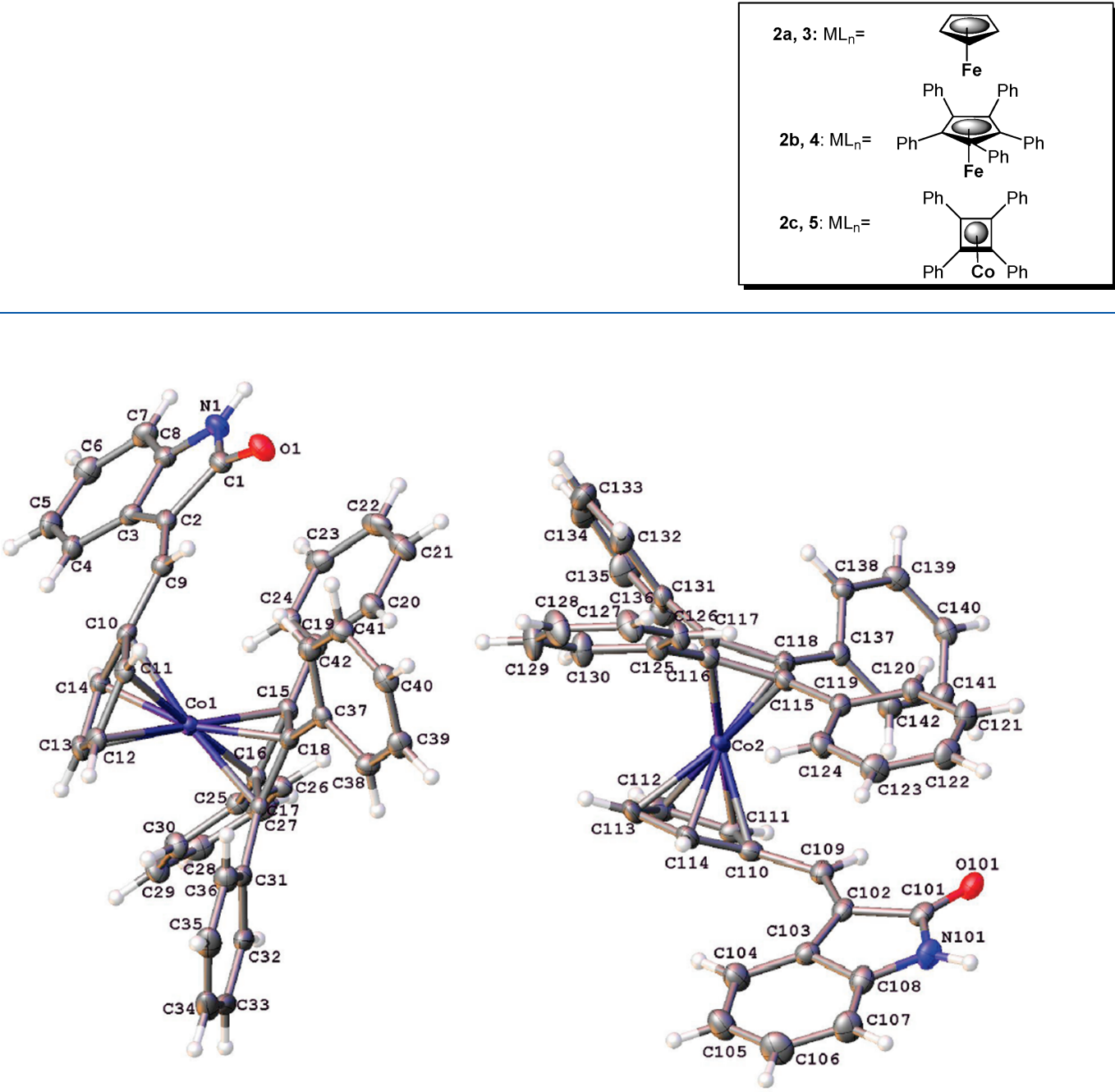

Figure 2. ADP plot of $(E)-5$ with ellipsoids shown at the $50 \%$ probability level.

commonplace. ${ }^{6}$ Microwave-mediated reactions of two sterically demanding aldehydes, namely, 1,2,3,4,5-pentaphenyl-ferrocene carboxaldehyde $(\mathbf{2 b})^{5 \mathrm{a}}$ and ( $\eta^{4}$-tetraphenyl-cyclobutadiene)$\left(\eta^{5}\right.$-cyclopentadienylcarboxaldehyde) cobalt $(2 \mathrm{c})^{5 \mathrm{~b}, \mathrm{c}}$ were carried out with 1 given our prior success in lowering reaction times and improving yields in the synthesis of 3 and analogues (Scheme 1). ${ }^{4}$ The air-stable products 4 and 5 were obtained as mixtures of geometrical isomers, which were separated by silica gel chromatography and assigned by ${ }^{1} \mathrm{H}$ NMR spectral comparison to our previously reported $(E)$ - and $(Z)-3 .{ }^{3}$ For example, the ${ }^{1} \mathrm{H}$ NMR spectrum of $(Z)-4$ showed a $C$ p signal, integrating as $2 \mathrm{H}$, at ca. $\delta=5.3 \mathrm{ppm}$, due to anisotropy of the adjacent $\mathrm{C}=\mathrm{O}$, whereas the corresponding E-isomer displayed the similarly attributable $2 \mathrm{H}$ at ca. $4.7 \mathrm{ppm}$. A similar trend was observed for $(Z)$ - and $(E)-5$ ( $\delta=5.7$ vs $5.1 \mathrm{ppm}$, respectively).

Solid-State Studies. Air-stable crystals of (E)- and (Z)5, suitable for single-crystal X-ray diffraction studies, were obtained from the diffusion of hexane into respective dichloromethane solutions. Both isomers crystallize in the space group $P 2_{1} / c$ (Figure $2, \mathrm{ADP}=$ atomic displacement parameters). The asymmetric unit of (E)-5 contains two molecules with similar geometries. Moreover, they are also similar to the structure of (Z)-5 and the other structures of this series (see Tables 2, 3 and Figures 2, 3). ${ }^{3,4}$ Thus, the bulky metallocene moieties in 
Table 1. Biological Activity of 3-5

$\begin{array}{ccccc} & \begin{array}{c}\mathrm{IC}_{50} \\ \text { compound } \\ (\mu \mathrm{M})^{a}\end{array} & \begin{array}{c}\text { PAK1 (\% residual } \\ \text { activity) }\end{array} & \begin{array}{c}\text { DYRK3 } \mathrm{IC}_{50} \\ (\mu \mathrm{M})\end{array} & \begin{array}{c}\mathrm{DYRK}^{2} \mathrm{IC}_{50} \\ (\mu \mathrm{M})\end{array} \\ \text { E-(3) } & 16 & 100^{b} & >10^{e} & 0.47^{e} \\ \mathrm{Z}-(3) & 4.3 & 100^{b} & 0.39^{e} & 1.1^{e} \\ E-(4) & >100 & 88^{c} & \text { n.d. } & \text { n.d. } \\ Z-(4) & >100 & 93^{c} & >10 & >10 \\ E-(5) & >100 & 100^{c} & \text { inactive }^{d} & \text { inactive }^{d} \\ Z-(5) & >100 & 83^{c} & \text { inactive }^{d} & \text { inactive }^{d}\end{array}$

${ }^{a}$ MTT assay, mean of two experiments; K562 (leukemia) cell line. ${ }^{b}$ At $10 \mu \mathrm{M}$ inhibitor concentration, \% residual activity in the presence of $1 \mu \mathrm{M}$ ATP. ${ }^{c}$ At $30 \mu \mathrm{M}$ inhibitor concentration, $\%$ residual activity in the presence of $1 \mu \mathrm{M} \mathrm{ATP} .{ }^{d}<10 \%$ inhibition at $10 \mu \mathrm{M}$. ${ }^{e}$ Values taken from ref 4.

Table 2. X-ray Crystallography Experimental Data

\begin{tabular}{|c|c|c|}
\hline & $(E)-5$ & $(Z)-5$ \\
\hline empirical formula & $\mathrm{C}_{42} \mathrm{H}_{30} \mathrm{CoNO}$ & $\mathrm{C}_{42} \mathrm{H}_{30} \mathrm{CoNO}$ \\
\hline $\mathrm{fw}$ & 623.60 & 623.60 \\
\hline$T / \mathrm{K}$ & $120(2)$ & $120(2)$ \\
\hline cryst syst & monoclinic & monoclinic \\
\hline space group & $P 2_{1} / c$ & $P 2_{1} / c$ \\
\hline$a / \AA ̊$ & $10.1505(2)$ & $11.3386(2)$ \\
\hline$b / \AA$ & $27.9585(6)$ & $26.6223(4)$ \\
\hline$c / \AA$ & $12.2065(2)$ & $21.0764(3)$ \\
\hline$\alpha / \operatorname{deg}$ & 90 & 90 \\
\hline$\beta / \operatorname{deg}$ & $106.8190(10)$ & $104.3580(10)$ \\
\hline$\gamma / \operatorname{deg}$ & 90 & 90 \\
\hline$V / \AA^{3}$ & $3315.93(11)$ & $6163.39(17)$ \\
\hline$D_{\text {calcd }} / \mathrm{g} \mathrm{cm}^{-3}$ & 1.249 & 1.344 \\
\hline \multirow[t]{2}{*}{ indep reflns } & 7571 & 14058 \\
\hline & {$\left[R_{\mathrm{int}}=0.0813\right]$} & {$\left[R_{\mathrm{int}}=0.0720\right]$} \\
\hline data/restraints/params & $7571 / 0 / 406$ & $14058 / 0 / 811$ \\
\hline absorp coeff $/ \mathrm{mm}^{-1}$ & 0.551 & 0.592 \\
\hline$R_{1} / w R_{2}\left(\right.$ observed data: $\left.F^{2}>2 \sigma\left(F^{2}\right)\right)$ & $0.0809 / 0.1484$ & $0.0668 / 0.1153$ \\
\hline$R_{1} / w R_{2}$ (all data) & $0.1152 / 0.1634$ & $0.0946 / 0.1277$ \\
\hline
\end{tabular}

(E)-5 and (Z)-5, compared with those of (E)-3 and (Z)-3, appear to have little effect on the geometries of the linker and oxindole units of the molecules. The largest difference between the molecular structures of $(E)-5$ and $(Z)-5$ is the planarity of the oxindole and metallocene units (torsion angles $\mathrm{C} 10-\mathrm{C} 9-\mathrm{C} 2-$ $\mathrm{C} 3=10.4(6)^{\circ}((E)-5), 1.1(4)^{\circ}((Z)-5) ; \mathrm{C} 110-\mathrm{C} 109-$ $\left.\left.\mathrm{C} 102-\mathrm{C} 103=11.5(6)^{\circ}(E)-5\right)\right)$. To some extent this difference is also observed between (E)-3 and (Z)-3. ${ }^{3}$ Intermolecular $\mathrm{H}$-bonded dimers of the type $\mathrm{C}=\mathrm{O} \cdots \mathrm{H}-\mathrm{N}$ are observed between the oxindole units in both isomers. The crystal structure of $(Z)-5$ contains solvent-accessible voids ( $532 \AA^{3}$ per unit cell) occupied by unidentified solvent molecules. A poor-quality crystal structure of $(Z)-4$, which proves connectivity but is not suitable for detailed analysis, was also obtained and is included in the Supporting Information, SI.

Biological Studies. Complexes $\mathbf{4}$ and $\mathbf{5}$ were evaluated against a number of kinases, namely, DYRK1-4 isoforms, HIPK4 (homeodomain-interacting protein kinase 1), JAK2 (Janus kinase-2), MAPKAPK3 (mitogen-activated protein kinaseactivated-3), and VEGFR2, which were previously shown to be

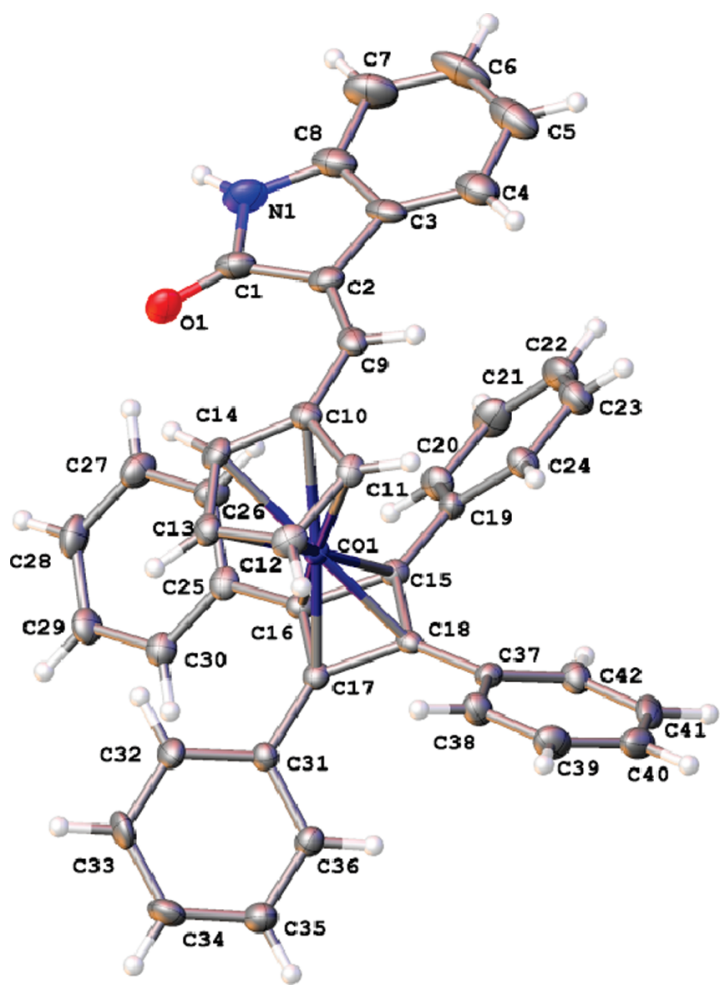

Figure 3. ADP plot of (Z)-5 with ellipsoids shown at the $50 \%$ probability level.

inhibited by 3 in the submicromolar to micromolar range. ${ }^{4}$ In all cases $\mathbf{4}$ and $\mathbf{5}$ showed no appreciable inhibition $(<10 \%$ inhibition at $10 \mu \mathrm{M}$ concentration), exemplified by DYRK3 and -4 (Table 1). Indeed, we next tested our complexes against PAK1 (p21-activated kinase-1), which is known to contain a large ATP binding domain and can accommodate large octahedral metal complexes. $^{7}$ At $10-30 \mu \mathrm{M}$ compound concentration, no inhibition was observed for $\mathbf{4}$ or $\mathbf{5}$ or even for $\mathbf{3}$. Moreover, $\mathbf{4}$ and $\mathbf{5}$ showed no appreciable effects on a leukemia cell line as opposed to 3 , which had moderate micromolar cytotoxicity (Table 1$){ }^{8}$

4 and 5 displayed no appreciable in vivo antiangiogenisis behavior in Xenopus embryos, which is in accordance with their in vitro screening results (Figure S2, SI).

\section{CONCLUSION}

Bulky oxindole-bound complexes 4 and 5 have been synthesized and tested for anticancer activity and were found to be inactive against a range of kinase targets compared with their simpler, less bulky ferrocene analog 3. Space-filling models emphasize the dramatic difference in the metal complex bulk around the common oxindole unit (Figure 4), which leads us to conclude that the bulky environment in $\mathbf{4}$ or $\mathbf{5}$ may disfavor binding in the ATP pocket of kinases, including that of PAK1.

\section{EXPERIMENTAL PROCEDURES}

Details on experimental and spectroscopic methods are as outlined previously. ${ }^{3}$ Reactions were carried out under argon using reagent grade solvents and reagents. X-ray structures have been deposited at the CCDC with the following codes: (E)-5, 818637; (Z)-5, 818638, and (Z)-4, 818639.

Synthesis of 4. 1,3-Dihydroindol-2-one (1) and $\eta^{5}$-1,2,3,4,5-pentaphenylferrocene carboxaldehyde (2b) were combined as described 
below for the synthesis of $\mathbf{5}$, except that toluene $(0.5 \mathrm{~mL})$ was used as a cosolvent with ethanol. ( $E$ )-4 (69\% yield) and $(Z)-4$ (30\% yield) were isolated after silica gel column chromatography using 9:1 dichloromethane/ethyl acetate as the elutant.

(E)-4: $\mathrm{mp} 200-202^{\circ} \mathrm{C}$ (solid crystallized from chloroform); IR (thin film) $v_{\max } 1600 \mathrm{~cm}^{-1}(\mathrm{C}=\mathrm{O}$ stretch $) ;{ }^{1} \mathrm{H} \operatorname{NMR}(\delta, 270.0 \mathrm{MHz}$, $\left.\mathrm{CDCl}_{3}\right)$; $4.61(2 \mathrm{H}, \mathrm{t}, J 1.83 \mathrm{~Hz}, \mathrm{Fc}), 4.75(2 \mathrm{H}, \mathrm{t}, J 1.83 \mathrm{~Hz}, \mathrm{Fc}), 6.85$ $(1 \mathrm{H}, \mathrm{d}, J 7.70 \mathrm{~Hz}, \mathrm{Ar}), 7.31(1 \mathrm{H}, \mathrm{td}, J 7.70,1.10 \mathrm{~Hz}, \mathrm{Ar}), 6.97-7.14$ (27H, m, $\operatorname{ArCH}), 7.20$ (1H, td, J 4.77, $1.10 \mathrm{~Hz}, \operatorname{ArCH}), 7.38(1 \mathrm{H}, \mathrm{s}$, $\mathrm{CH}) ;{ }^{13} \mathrm{C}$ NMR $\left(\delta, 67.0 \mathrm{MHz}, \mathrm{CDCl}_{3}\right) 76.6,78.6,81.7,88.5,109.6$, $109.9,121.6,122.2$, 122.4, 122.9, 124.6, 126.2, 126.4, 126.9, 127.2, 127.9, $128.8,132.2,134.7,137.0,141.2,169.5$; HRMS $(m / z$, HNESP) for $[\mathrm{M}+\mathrm{H}]^{+}$for $\mathrm{C}_{49} \mathrm{H}_{36} \mathrm{FeNO}$ calcd 710.2146 , obsd 710.2129.

(Z)-4: mp 180-182 ${ }^{\circ} \mathrm{C} ;{ }^{1} \mathrm{H} \mathrm{NMR}\left(\delta, 270.0 \mathrm{MHz}, \mathrm{CDCl}_{3}\right) 4.62(2 \mathrm{H}$, t, J $1.83 \mathrm{~Hz}, \mathrm{Fc}), 5.26(2 \mathrm{H}, \mathrm{t}, J 1.83 \mathrm{~Hz}, \mathrm{Fc}), 6.74(1 \mathrm{H}, \mathrm{d}, J 8.06 \mathrm{~Hz}, \mathrm{Ar})$, $7.31(1 \mathrm{H}, \mathrm{td}, J 7.70,1.10 \mathrm{~Hz}, \mathrm{Ar}), 6.85-7.18(28 \mathrm{H}, \mathrm{m}, \mathrm{ArCH}), 7.54(1 \mathrm{H}$, s, $\mathrm{CH}) ;{ }^{13} \mathrm{C} \mathrm{NMR}\left(\delta, 67.0 \mathrm{MHz}, \mathrm{CDCl}_{3}\right) 78.0,80.7,88.4,109.5,118.4$, $121.4,125.1,125.4,126.3,127.1,127.7,132.2,135.00,137.2,138.5$, 169.6; HRMS $\left(m / z\right.$, HNESP) for $[\mathrm{M}+\mathrm{H}]^{+}$for $\mathrm{C}_{49} \mathrm{H}_{36} \mathrm{FeNO}$ calcd 710.2146 , obsd 710.2128 .

Table 3. Selected Bond Lengths (̊̊), Angles (deg), and Torsion Angles (deg) for (E)-5 and (Z)-5

\begin{tabular}{|c|c|c|c|}
\hline & \multicolumn{2}{|c|}{$(E)-5$} & $(Z)-5$ \\
\hline $\mathrm{C} 9-\mathrm{C} 10$ & $1.454(4)$ & & $1.454(6)$ \\
\hline $\mathrm{C} 9-\mathrm{C} 2$ & $1.349(4)$ & & $1.356(6)$ \\
\hline $\mathrm{C} 1-\mathrm{C} 2$ & $1.495(4)$ & & $1.492(6)$ \\
\hline $\mathrm{C} 2-\mathrm{C} 3$ & $1.471(4)$ & & $1.459(6)$ \\
\hline $\mathrm{C} 1-\mathrm{N} 1$ & $1.363(4)$ & & $1.361(5)$ \\
\hline $\mathrm{N} 1-\mathrm{C} 8$ & $1.399(4)$ & & $1.397(6)$ \\
\hline $\mathrm{O} 1-\mathrm{C} 1$ & $1.234(4)$ & & $1.226(5)$ \\
\hline $\mathrm{C} 109-\mathrm{C} 110$ & & $1.449(4)$ & \\
\hline $\mathrm{C} 109-\mathrm{C} 102$ & & $1.345(4)$ & \\
\hline $\mathrm{C} 101-\mathrm{C} 102$ & & $1.502(4)$ & \\
\hline $\mathrm{C} 102-\mathrm{C} 103$ & & $1.470(4)$ & \\
\hline $\mathrm{C} 101-\mathrm{N} 101$ & & $1.363(4)$ & \\
\hline $\mathrm{N} 101-\mathrm{C} 108$ & & $1.399(4)$ & \\
\hline $\mathrm{O} 101-\mathrm{C} 101$ & & $1.235(4)$ & \\
\hline $\mathrm{C} 2-\mathrm{C} 9-\mathrm{C} 10$ & $131.8(3)$ & & $133.4(4)$ \\
\hline $\mathrm{C} 1-\mathrm{N} 1-\mathrm{C} 8$ & $110.9(2)$ & & $111.5(4)$ \\
\hline $\mathrm{O} 1-\mathrm{C} 1-\mathrm{N} 1$ & $125.4(3)$ & & $124.3(4)$ \\
\hline $\mathrm{C} 102-\mathrm{C} 109-\mathrm{C} 110$ & & $130.8(3)$ & \\
\hline $\mathrm{C} 101-\mathrm{N} 101-\mathrm{C} 108$ & & $110.9(3)$ & \\
\hline $\mathrm{O} 101-\mathrm{C} 101-\mathrm{N} 101$ & & $125.8(3)$ & \\
\hline $\mathrm{C} 10-\mathrm{C} 9-\mathrm{C} 2-\mathrm{C} 3$ & $10.4(6)$ & & $1.1(4)$ \\
\hline $\mathrm{C} 110-\mathrm{C} 109-\mathrm{C} 102-\mathrm{C} 103$ & & $11.5(6)$ & \\
\hline
\end{tabular}

$(E) /(Z)-4$ mixture: Anal. Calcd for $\mathrm{C}_{49} \mathrm{H}_{35} \mathrm{FeNO} \cdot 0.4$ chloroform: C, 78.20, H, 4.84, N, 1.85. Found: C, 78.27, H, 4.44, N, 1.53 .

General Microwave Method. Synthesis of $\mathbf{5}$. In an oven-dried microwave tube $(35 \mathrm{~mL})$ equipped with a stirrer, 1,3-dihydroindol-2one (1) $(0.013 \mathrm{~g}, 0.09 \mathrm{mmol})$ and $\left(\eta^{4}\right.$-tetraphenylcyclobutadiene $)\left(\eta^{5}\right.$ cyclopentadienylcarboxaldehyde)cobalt (2c) $(0.048 \mathrm{~g}, 0.09 \mathrm{mmol})$ were dissolved in ethanol $(2.5 \mathrm{~mL})$ and piperidine $(0.25 \mu \mathrm{L})$. The reaction mixture was allowed to stir for 2 min before being irradiated using a microwave for $30 \mathrm{~min}$ at $150 \mathrm{~W}$ and $150^{\circ} \mathrm{C}$. After cooling, solvents were removed under reduced pressure using a rotary evaporator. The crude reaction mixture was given an aqueous workup, diluted first with ethyl acetate $(10 \mathrm{~mL})$ and then washed with distilled water $(10 \mathrm{~mL})$ and brine $(2 \times 10 \mathrm{~mL}$, sat.). The organic layer was dried using magnesium sulfate and filtered using fluted filter paper. The filtrate was then concentrated in vacuo. (E)-5 (0.03 g, 54\%) and (Z)-5 (0.025 g, 44\%) were isolated after silica gel column chromatography using 19:1 hexane/ethyl acetate as the eluant.

(E)-5: mp 198-200 ${ }^{\circ} \mathrm{C} ;{ }^{1} \mathrm{H} \mathrm{NMR}\left(\delta, 270.0 \mathrm{MHz}, \mathrm{CDCl}_{3}\right) 4.94(2 \mathrm{H}$, t, J $\left.1.83 \mathrm{~Hz}, \mathrm{C}_{5} \mathrm{H}_{4}\right), 5.15\left(2 \mathrm{H}, \mathrm{t}, J 1.83 \mathrm{~Hz}, \mathrm{C}_{5} \mathrm{H}_{4}\right), 6.80-6.92(2 \mathrm{H}, \mathrm{m}$, $\mathrm{Ar}), 7.00(1 \mathrm{H}, \mathrm{s},=\mathrm{CH}), 7.12-7.24(13 \mathrm{H}, \mathrm{m}, \mathrm{Ar}), 7.37-7.40(8 \mathrm{H}, \mathrm{m}$, $\mathrm{Ar}), 7.51-7.64(2 \mathrm{H}, \mathrm{m}, \mathrm{Ar}) ;{ }^{13} \mathrm{C} \mathrm{NMR}\left(\delta, 67.0 \mathrm{MHz}, \mathrm{CDCl}_{3}\right) 80.5$, 82.9, 105.2, 107.9, 117.1, 117.7, 117.9, 118.6, 119.4, 120.2, 121.3, 122.2, 123.7, 124.3, 129.7, 130.6, 134.2, 165.8; HRMS $(m / z$, HNESP) for $\mathrm{C}_{42} \mathrm{H}_{31} \mathrm{CoNO}[\mathrm{M}+\mathrm{H}]^{+}$calcd 624.1738, obsd 624.1724.

(Z)-5: mp 214-216 ${ }^{\circ} \mathrm{C} ;{ }^{1} \mathrm{H}$ NMR $\left(\delta, 270.0 \mathrm{MHz}, \mathrm{CDCl}_{3}\right) 4.91(2 \mathrm{H}$, t, J $\left.1.83 \mathrm{~Hz}, \mathrm{C}_{5} \mathrm{H}_{4}\right), 5.71\left(2 \mathrm{H}, \mathrm{t}, J 1.83 \mathrm{~Hz}, \mathrm{C}_{5} \mathrm{H}_{4}\right), 6.61(1 \mathrm{H}, \mathrm{s},=\mathrm{CH})$, $6.77(1 \mathrm{H}, \mathrm{d}, J 6.48 \mathrm{~Hz}, \mathrm{Ar}), 6.90-6.96(2 \mathrm{H}, \mathrm{m}, \mathrm{Ar}), 7.08-7.20(14 \mathrm{H}, \mathrm{m}$, $\mathrm{Ar}), 7.36-7.40(8 \mathrm{H}, \mathrm{m}, \mathrm{Ar}) ;{ }^{13} \mathrm{C} \mathrm{NMR}\left(\delta, 67.0 \mathrm{MHz}, \mathrm{CDCl}_{3}\right) 86.9$, 87.8, 90.5, 109.0, 118.4, 121.3, 122.9,125.6, 126.5, 127.4, 128.1, 128.7, 133.2, 135.4, 138.5, 167.8; HRMS $\left(m / z\right.$, HNESP) for $\mathrm{C}_{42} \mathrm{H}_{31} \mathrm{CoNO}$ $[\mathrm{M}+\mathrm{H}]^{+}$calcd 624.1738 , obsd 624.1725.

$(E) /(Z)-5$ mixture: Anal. Calcd for $\mathrm{C}_{42} \mathrm{H}_{30} \mathrm{CoNO}$ : C, 80.89, $\mathrm{H}, 4.85$, N, 2.25. Found: C, 80.96, H, 4.93, N, 2.32 .

X-ray Crystallography. Single-crystal X-ray diffraction analyses of $(E)-5$ and $(Z)-5$ were performed using a Bruker APEXII CCD diffractometer mounted at the window of a Bruker FR591 rotating anode (Mo K $\alpha=0.71073 \AA$ ) and equipped with an Oxford Cryosystems cryostream device (SI, Table S1). Data were processed using the Collect package, ${ }^{9}$ and unit cell parameters were refined against all data. An empirical absorption correction was carried out using SADABS. ${ }^{10}$ The structures were solved by either direct methods using SHELXS $-97^{11}$ or charge-flipping using SUPERFLIP ${ }^{12}$ and refined on $F_{\mathrm{o}}{ }^{2}$ by full-matrix least-squares refinements using SHELXL-97. ${ }^{13}$ All non-hydrogen atoms were refined with anisotropic displacement parameters. All hydrogen atoms were added at calculated positions and refined using a riding model with isotropic displacement parameters based on the equivalent isotropic displacement parameter $\left(U_{\text {eq }}\right)$ of the parent atom. Figures were produced using OLEX2 ${ }^{14}$ and MERCURY. ${ }^{15}$ The $(Z)-5$ structure contains two solvent-accessible voids per unit cell (216 $\AA^{3}$ each), which were investigated using PLATON SQUEEZE. ${ }^{16}$



Figure 4. Molecular structures of $(Z)-3,(Z)-4$, and $(Z)-5$. The units common to all structures are shown in ball-and-stick representation, while the differences in the metallocene substituents are shown in space-filling representation (see SI). 


\section{ASSOCIATED CONTENT}

S Supporting Information. This material is available free of charge via the Internet at http://pubs.acs.org.

\section{AUTHOR INFORMATION}

Corresponding Author

*Fax: +44 (0)2083319805. E-mail: j.spencer@gre.ac.uk.

\section{ACKNOWLEDGMENT}

The Royal Society of Chemistry is thanked for a Research Fund (J.S.); the EPSRC Mass Spectrometry Service (University of Swansea) is thanked for HRMS measurements. J.A.H. acknowledges support from CRUK (C2259/A9994). J.S. acknowledges support from Greenwich University and the Alumni Fund.

\section{REFERENCES}

(1) Reviews: (a) Hartinger, C. G.; Dyson, P. J. Chem. Soc. Rev. 2009, 38, 391. (b) Hillard, E. A.; Jaouen, G. Organometallics 2011, 30, 20. (c) Meggers, E. Chem. Commun. 2009, 1001. (d) van Rijt, S. H.; Sadler, P. Drug Discovery Today 2010, 14, 1089. (e) Gasser, G.; Ott, I.; Metzler-Nolte, N. J. Med. Chem. 2011, 54, 3.

(2) (a) Wilbuer, A.; Vlecken, D. H.; Schmitz, D. J.; Kräling, K.; Harms, K.; Bagowski, C. P.; Meggers, E. Angew. Chem., Int. Ed. 2010, 49, 3839. (b) Atilla-Gokcumen, G. E.; Williams, D. S.; Bregman, H.; Pagano, N.; Meggers, E. Chembiochem 2006, 7, 1443. (c) Bullock, A. N.; Russo, S.; Amos, A.; Pagano, N.; Bregman, H.; Debreczeni, J. E.; Lee, W. H.; von Delft, F.; Meggers, E.; Knapp, S. PLoS One 2009, 4 (10), e7112.

(3) Spencer, J.; Mendham, A. P.; Kotha, A. K.; Richardson, S. C.; Hillard, E. A.; Jaouen, G.; Male, L.; Hursthouse, M. B. Dalton Trans. 2009, 918.

(4) Spencer, J.; Amin, J.; Callear, S. K.; Tizzard, G. J.; Coles, S. J.; Coxhead, P.; Guille, M. Metallomics 2011. DOI: 10.1039/c1mt00017a.

(5) (a) Butler, D. C. D.; Richards, C. J. Organometallics 2002, 21, 5433. (b) Rausch, M. D.; Genetti, R. D. J. Org. Chem. 1970, 35, 3888. (c) Stevens, A. M.; Richards, C. J. Tetrahedron Lett. 1997, 38, 7805.

(6) Microwave-mediated organometallic synthesis: (a) Albrecht, C.; Gauthier, S.; Wolf, J.; Scopelliti, R.; Severin, K. Eur. J. Inorg. Chem. 2009, 8, 967. Microwave-mediated synthesis of kinase inhibitors see: (b) Bagley, M. C.; Davis, T.; Dix, M. C.; Murziani, P. G.; Rokicki, M. J.; Kipling, D. Bioorg. Med. Chem. Lett. 2008, 18, 3745.

(7) Maksimoska, J.; Feng, Li.; Harms, K.; Yi, C.; Kissil, J.; Marmorstein, R.; Meggers, E. J. Am. Chem. Soc. 2008, 130, 15764.

(8) For previous biological assay details see: Urbaniak, M. D.; Bingham, J. P.; Hartley, J. A.; Woolfson, D. N.; Caddick, S. J. Med. Chem. 2004, 47, 4710.

(9) Hooft, R.; Nonius, B. V. Collect: Data Collection Software; B.V. Nonius, 1998.

(10) Sheldrick, G. M. SADABS, Version 2.10; Bruker AXS Inc.: Madison, WI, USA, 2003.

(11) Sheldrick, G. M. Acta Crystallogr. 2008, A64, 112.

(12) Palatinus, L.; Chapuis, G. J. Appl. Crystallogr. 2007, 40, 786.

(13) Dolomanov, O. V.; Bourhis, L. J.; Gildea, R. J.; Howard, J. A. K.; Puschmann, H. J. Appl. Crystallogr. 2009, 42, 339.

(14) Macrae, C. F.; Bruno, I. J.; Chisholm, J. A.; Edgington, P. R.; McCabe, P.; Pidcock, E.; Rodriguez-Monge, L.; Taylor, R.; van de Streek, J.; Wood, P. A. J. Appl. Crystallogr. 2008, 41, 466.

(15) Sluis, P. V.D.; Spek, A. L. Acta Crystallogr., Sect. A 1990, 46, 194.

(16) Macrae, C. F.; Bruno, I. J.; Chisholm, J. A.; Edgington, P. R.; McCabe, P.; Pidcock, E.; Rodriguez-Monge, L.; Taylor, R.; van de Streek, J.; Wood, P. A. J. Appl. Crystallogr. 2008, 41, 466. 